\title{
DERECHO Y POLÍTICAS AMBIENTALES EN LA COMUNIDAD DE MADRID
}

(PRIMER SEMESTRE 2020)

\author{
DANiel B. Entrena Ruiz \\ Profesor Contratado Doctor \\ Universidad Carlos III de Madrid
}


Sumario: 1 . orden $367 / 2020$, de 13 de marzo, de la Consejería de Sanidad, por la que se adoptan medidas preventivas de salud pública en la Comunidad de Madrid como consecuencia de la situación y evolución del coronavirus (COVID-19). 2. Decreto 316/2019, de 27 de diciembre, del Consejo de Gobierno, por el que se modifica el Decreto 278/2019, de 29 de octubre, por el que se establece la estructura orgánica de la Consejería de Medio Ambiente, Ordenación del Territorio y Sostenibilidad. 3. Decreto 18/2020, de 11 de febrero, del Consejo de Gobierno, por el que se aprueba el Plan Rector de Uso y Gestión del Parque Nacional de la Sierra de Guadarrama en el ámbito territorial de la Comunidad de Madrid. 4. Resolución de 4 de febrero de 2020, del Director General de Biodiversidad y Recursos Naturales, de la Consejería de Medio Ambiente, Ordenación del Territorio y Sostenibilidad, por la que se regula el tránsito por viales, el tránsito en las inmediaciones y pie de vía de sectores de escalada y la escalada en los sectores y vías regulados, en el paraje de La Pedriza del Parque Nacional de la Sierra de Guadarrama como medida de protección de especies rupícolas durante su época de cría y reproducción. 5 Orden 468/2020, de 24 de febrero, de la Consejería de Medio Ambiente, Ordenación del Territorio y Sostenibilidad, sobre establecimiento de vedas y regulación especial de la actividad piscícola en los ríos, arroyos y embalses de la Comunidad de Madrid para la temporada 2020

1. ORDEN 367/2020, DE 13 DE MARZO, DE LA CONSEJERÍA DE SANIDAD, POR LA QUE SE ADOPTAN MEDIDAS PREVENTIVAS DE SALUD PÚBLICA EN LA COMUNIDAD DE MADRID COMO CONSECUENCIA DE LA SITUACIÓN Y EVOLUCIÓN DEL CORONAVIRUS (COVID-19).

Después de todo lo acontecido en relación con esta plaga, seguramente haya quedado desactualizada el contenido de la Orden que referenciamos. A pesar de ello, no está de más rememorarla para recordar la difícil etapa vivida y cómo se comenzó a actuar en este caso desde la Comunidad de Madrid antes de la formal adopción del estado de alarma por Real Decreto 463/2020, de 14 de marzo, adelantándose a su contenido.

De ella vamos a referir los preceptos que invoca para su cobertura, las medidas previamente adoptadas y, finalmente, las que incorporó.

Así, la referida Orden invoca para su aprobación, en primer lugar, la Ley Orgánica 3/1986, de 14 de abril, de Medidas Especiales en Materia de Salud Pública, de conformidad con la cual (artículo 2):

"las autoridades sanitarias competentes podrán adoptar medidas de reconocimiento, tratamiento, hospitalización o control cuando se aprecien indicios racionales que permitan suponer la existencia de peligro para la salud de la población debido a la situación sanitaria concreta de una persona o grupo de personas o por las condiciones sanitarias en que se desarrolle una actividad". 
Asimismo, su artículo 3 indica: "con el fin de controlar las enfermedades transmisibles, la autoridad sanitaria, además de realizar las acciones preventivas generales, podrá adoptar las medidas oportunas para el control de los enfermos, de las personas que estén o hayan estado en contacto con los mismos y del medio ambiente inmediato, así como las que se consideren necesarias en caso de riesgo de carácter transmisible".

La segunda invocación que efectúa la Orden es el artículo veintiséis de la Ley 14/1986, de 25 de abril, General de Sanidad:

“1. En caso de que exista o se sospeche razonablemente la existencia de un riesgo inminente y extraordinario para la salud, las autoridades sanitarias adoptarán las medidas preventivas que estimen pertinentes, tales como la incautación o inmovilización de productos, suspensión del ejercicio de actividades, cierres de Empresas o sus instalaciones, intervención de medios materiales y personales y cuantas otras se consideren sanitariamente justificadas.

2. La duración de las medidas a que se refiere el apartado anterior, que se fijarán para cada caso, sin perjuicio de las prórrogas sucesivas acordadas por resoluciones motivadas, no excederá de lo que exija la situación de riesgo inminente y extraordinario que las justificó.”

Tras ello, la Orden alude a la Ley 33/2011, de 4 de octubre, General de Salud Pública, que en su artículo 54 prevé que con carácter excepcional y cuando así lo requieran motivos de extraordinaria gravedad o urgencia, la autoridad competente de las comunidades autónomas puede adoptar, mediante resolución motivada, entre otras medidas la suspensión del ejercicio de actividades.

Finalmente, se menciona el artículo 55.1 de la Ley 12/2001, de 21 de diciembre, de Ordenación Sanitaria de la Comunidad de Madrid que dispone que la función de Autoridad en Salud Pública incluye: “a) La adopción, cuando proceda, de las medidas previstas en la Ley 3/1986, de 14 de abril, de Medidas Especiales en Materia de Salud Pública, y especialmente en los supuestos contemplados en sus artículos 2 y 3. b) La adopción de cualquier otra medida necesaria en función del análisis de los determinantes del proceso salud-enfermedad en la Comunidad de Madrid". 
Por lo que respecta a las medidas ya desarrolladas en relación con el COVID19 , la Orden que referenciamos menciona las adoptadas en:

i. centros de mayores, en las tipologías de hogares y clubes y servicios de convivencia familiar y social y actividades formativas de los centros sanitarios (Resoluciones de 6 de marzo de 2020 de la Dirección General de Salud Pública de la Comunidad de Madrid)

ii. ámbito docente y en materia de transporte regular de viajeros (Orden 338/2020, de 9 de marzo, de la Consejería de Sanidad)

iii. ámbito deportivo, actividades colectivas, cursos de formación y Bibliotecas Públicas de la Comunidad de Madrid (Orden 344/2020, de 10 de marzo)

iv. actividad física y cultural (Orden 348/2020, de 11 de marzo, de la Consejería de Sanidad)

v. actividad de centros ocupacionales que prestan atención a personas con discapacidad intelectual y los servicios sociales de atención temprana a menores (Resolución de 11 de marzo de 2020, de la Dirección General de Salud Pública de la Comunidad de Madrid)

La Orden que ahora referenciamos adopta nuevas medidas a iniciativa de distintas Consejerías, en particular de distanciamiento social en el ámbito de sus respectivas competencias, consistentes sucintamente en:

i) suspensión de espectáculos públicos, actividades recreativas, establecimientos, locales e instalaciones;

ii) suspensión de la actividad presencial en la Oficina de Atención al Ciudadano de la Comunidad de Madrid y Oficinas de Asistencia en Materia de Registro de la Administración de la Comunidad de Madrid, así como en los Puntos de Información y Atención al Ciudadano y Oficinas de Información Especializada de la Comunidad de Madrid.

iii) suspensión de la actividad comercial minorista en todo el territorio de la Comunidad de Madrid, a excepción de los establecimientos comerciales minoristas de alimentación y productos y bienes de primera necesidad recogidos a continuación según la clasificación nacional de actividades económicas 2009 (CNAE-2009) 
iv) suspensión de la actividad formativa presencial en los centros que impartan enseñanzas no regladas en el ámbito territorial de la Comunidad de Madrid.

v) suspensión de la actividad y de la apertura al público de locales de ensayo, centros de formación, de usos múltiples, con espacios de biblioteca, salas de lectura y archivos de documentación, albergues, refugios juveniles y servicios de información juvenil.

vi) suspensión del ejercicio de actividades de los centros de atención diurna a personas mayores y a personas con discapacidad ubicados en el territorio de la Comunidad de Madrid. No obstante, en este caso se excepcionaron los centros que debieran garantizar la atención a aquellas personas mayores que, por su especial situación de vulnerabilidad, requirieses una atención especializada que no pudiera prestarse en su domicilio.

2. DECRETO 316/2019, DE 27 DE DICIEMBRE, DEL CONSEJO DE GOBIERNO, POR EL QUE SE MODIFICA EL DECRETO 278/2019, DE 29 DE OCTUBRE, POR EL QUE SE ESTABLECE LA ESTRUCTURA ORGÁNICA DE LA CONSEJERÍA DE MEDIO AMBIENTE, ORDENACIÓN DEL TERRITORIO Y SOSTENIBILIDAD

Tras la celebración de elecciones autonómicas, mediante Decreto 52/2019, de 19 de agosto, de la Presidenta de la Comunidad de Madrid, se determinó el número y denominación de las Consejerías, incluyendo la creación de la Consejería de Medio Ambiente, Ordenación del Territorio y Sostenibilidad, a la que atribuía las competencias hasta desarrolladas por la Consejería de Medio Ambiente y Ordenación del Territorio.

Poco después, el Decreto 73/2019, de 27 de agosto, del Consejo de Gobierno, modificó la estructura orgánica básica de las Consejerías de la Comunidad de Madrid y, a consecuencia de ello, el Decreto 278/2019, de 29 de octubre, estableció la estructura orgánica de la Consejería de Medio Ambiente, Ordenación del Territorio y Sostenibilidad.

Sin embargo, la distribución competencial realizada por este último Decreto 278/2019, de 29 de octubre, no era coincidente de forma parcial con la 
modificación efectuada por el Decreto 73/2019, de 27 de agosto; en particular respecto al ejercicio de la potestad sancionadora, que este último Decreto atribuye de manera a la Dirección General de Economía Circular. El Decreto ahora referenciado atribuye en el desarrollo de la estructura orgánica básica a este órgano administrativo, las competencias en materia de inspección y disciplina ambiental y, en dependencia de él, a la Subdirección General de Inspección y Disciplina Ambiental.

Además, el Decreto clarifica y concreta también otras competencias de este mismo órgano a la que se confiere "La promoción y difusión de los principios y objetivos de la economía circular", las relativas a calidad ambiental y economía circular.

Finalmente, el Decreto también actualiza la denominación de la Subdirección General de Residuos, pues al tener atribuidas funciones en materia de calidad hídrica, ámbito éste que se incorpora expresamente a su denominación.

\section{DECRETO 18/2020, DE 11 DE FEBRERO, DEL CONSEJO DE GOBIERNO, POR EL QUE SE APRUEBA EL PLAN RECTOR DE USO Y GESTIÓN DEL PARQUE NACIONAL DE LA SIERRA DE GUADARRAMA EN EL ÁMBITO TERRITORIAL DE LA COMUNIDAD DE MADRID}

Los órganos legislativos de la Comunidad de Madrid y de la Junta de Castilla y León impulsaron la creación del Parque Nacional de la Sierra de Guadarrama, que vio la luz mediante la Ley $7 / 2013$, de 25 de junio.

Desde entonces, desaparecieron los espacios protegidos sobre los que se ha declarado el parque nacional: El Parque Natural de Peñalara, Cumbre, Circo y Lagunas, que queda completamente comprendido por el parque nacional, y el Parque Regional de la Cuenca Alta del Manzanares, incluido solo en parte de su ámbito territorial.

El Parque Nacional de la Sierra de Guadarrama cuenta con una superficie de 33.960 hectáreas pertenecientes a las comunidades autónomas de Madrid (21.714 hectáreas) y de Castilla y León (12.246 hectáreas), correspondiendo la gestión ordinaria y habitual de este espacio a ambas comunidades en sus 
respectivos ámbitos territoriales, debiendo asegurarse la gestión integral del parque nacional.

De conformidad con el artículo 20 de la Ley 30/2014, de 3 de diciembre, de Parques Nacionales, las Comunidades Autónomas implicadas deben aprobar el correspondiente Plan Rector de Uso y Gestión, lo que se lleva al efecto en el ámbito de Madrid mediante el Decreto que referenciamos, de acuerdo con su vez con el Real Decreto 389/2016, de 22 de octubre, por el que se aprueba el Plan Director de la Red de Parques Nacionales.

El Plan Rector de Uso y Gestión establece los objetivos de la planificación, los criterios de gestión y la zonificación del parque. Asimismo, recoge la relación de las actividades clasificadas en incompatibles, compatibles y, entre estas, las específicamente necesarias para la gestión, así como las condiciones bajo las que pueden desarrollarse las actividades compatibles con los objetivos del parque nacional.

Por ejemplo, se declaran incompatibles -y por tanto prohibidas- entre otras las siguientes (artículos 38, 41): la acampada libre para permanecer y pernoctar de tiendas de campaña, caravanas u otros elementos de acampada fijos o móviles, incluyendo vehículos o remolques de cualquier tipo; las pruebas y competiciones deportivas, salvo aquellas de baja incidencia ambiental; la actividad hostelera, la venta ambulante, a celebración de conciertos musicales, fiestas multitudinarias y otros eventos similares. Sin embargo, algunas de esas prohibiciones son excepcionadas, para permitirlas bajo condiciones y lugares que el propio Plan especifica.

El Plan también integra las directrices para la elaboración de los programas sectoriales de actuación, donde se deben recoger las medidas necesarias para proteger y conservar los valores naturales y culturales del parque, que comprenderán, entre otras, las actuaciones precisas para la consecución de los objetivos del parque nacional en materias tales como conservación, uso público, desarrollo socioeconómico, investigación y educación ambiental, así como la estimación económica de las inversiones correspondientes.

En particular, deben desarrollarse dichos Programas sectoriales en las siguientes áreas: a) Conservación de los recursos naturales, patrimonio cultural 
y paisaje; b) Investigación; c) Uso Público y Social; d) Relaciones con el entorno y participación ciudadana; e) Seguimiento, vigilancia y control.

4. RESOLUCIÓN DE 4 DE FEBRERO DE 2020, DEL DIRECTOR GENERAL DE BIODIVERSIDAD Y RECURSOS NATURALES, DE LA CONSEJERÍA DE MEDIO AMBIENTE, ORDENACIÓN DEL TERRITORIO Y SOSTENIBILIDAD, POR LA QUE SE REGULA EL TRÁNSITO POR VIALES, EL TRÁNSITO EN LAS INMEDIACIONES Y PIE DE VÍA DE SECTORES DE ESCALADA Y LA ESCALADA EN LOS SECTORES Y VÍAS REGULADOS, EN EL PARAJE DE LA PEDRIZA DEL PARQUE NACIONAL DE LA SIERRA DE GUADARRAMA COMO MEDIDA DE PROTECCIÓN DE ESPECIES RUPÍCOLAS DURANTE SU ÉPOCA DE CRÍA Y REPRODUCCIÓN

En cumplimiento de lo previsto en el Plan Rector de Uso y Gestión, se aprueba mediante la resolución indicada regula la escalada y tránsito en sus inmediaciones en el Parque Nacional de la Sierra de Guadarrama. Una actividad de gran tradición en la zona, hasta el punto de convertirla en un referente de fama internacional.

Sin embargo, al desarrollarse en ámbitos de alta relevancia ambiental y ante el incremento del número de practicantes, se hace necesario su regulación, que asegure el disfrute de esta actividad de forma que no se alteren los principios que rigen la gestión de un Parque Nacional.

En particular para evitar daños donde existen nidos de aves, concretamente de buitre leonado (Gyps fulvus), halcón peregrino (Falco peregrinus) y águila real (Aquila chrysaetos) y la necesidad de conservar estas aves en el período de cría.

Pero, además, dicha regulación busca evitar posibles deterioros en la roca, daños en la base de la pared o en los senderos de acceso, fruto de la presencia de un gran número de escaladores.

Por ello, se establece una zonificación, incluyendo aquellas en las que se restringe temporalmente la escalada por la presencia de nidos; unas en las que sólo se restringe la escalada por vías concretas y otras en las que por afectar a especies más sensibles se requiere la restricción temporal de todo el sector. 
Finalmente, la resolución regula el tránsito en ciertos viales que, por su proximidad a nidos, pudieran ocasionar molestias.

Fuera de esas zonas y períodos concretos, la resolución permite la práctica de la escalada, el búlder y el rápel, bajo el régimen general que pudieran afectar a estas actividades.

\section{ORDEN 468/2020, DE 24 DE FEBRERO, DE LA CONSEJERÍA DE MEDIO} AMBIENTE, ORDENACIÓN DEL TERRITORIO Y SOSTENIBILIDAD, SOBRE ESTABLECIMIENTO DE VEDAS Y REGULACIÓN ESPECIAL DE LA ACTIVIDAD PISCÍCOLA EN LOS RÍOS, ARROYOS Y EMBALSES DE LA COMUNIDAD DE MADRID PARA LA TEMPORADA 2020

Finalmente, pese a la suspensión de toda actividad pesquera recreativa declarada en nuestro país a consecuencia del COVID-19, la Consejería de Medio Ambiente, Ordenación del Territorio y Sostenibilidad regula el aprovechamiento de las masas de agua de la Comunidad de Madrid.

Esta Orden regula así el régimen de los permisos de pesca y tasas aplicables, en atención a su vez a los distintos tipos de pescadores (integrantes de sociedad que gestionan cotos, "ribereños" -esto es, residentes en municipios próximos a los cotos- y resto de pescadores), las especies que pueden ser objeto de pesca (dimensiones mínimas y cupos), régimen de la actividad pesquera y zonas donde puede desarrollarse y los periodos hábiles en 2020, comercialización, y régimen sancionador, por referir mínimamente su contenido. 